\title{
An Efficient Speckle Noise Reduction Filter in Ultrasound images using Discrete Wavelet Transform based on Adaptive Threshold
}

\author{
K.M. Aslam Uddin, Md. Masudur Rahman \\ ICE Department, Noakhali Science and Technology University, Bangladesh
}

\begin{abstract}
Noise is the major problem in the field of image processing. In Medical image such as Ultrasound image are contaminated by different types of noise. The usefulness of US imaging is degraded by the presence of signal dependence noise known as speckle noise. In the presence of such noise it is difficult to diagnosis. To acquire a better performance we state another method that works efficiently to reduce noise an image without blurring the frontiers between different regions. This paper demonstrates wavelet based thresholding technique for de-noising and improving visual image quality in ultrasound images. This proposed method is compared to other existing approach and give superior result that satisfy the human visual quality and also these resulting images are evaluated by the performance parameter Signal to Noise Ratio, The Peak Signal to Noise Ratio and also other image quality measurement criteria. This method gives the better result with comparison to existing the Adaptive filter and Anisotropic Diffusion filter.
\end{abstract}

Keywords: US images, Speckle noise, dwt, threshold

\section{Introduction}

Ultrasound imaging is one of the most widely used diagnostic tools in modern medicine. The technology is relatively inexpensive and portable, especially when compared with other imaging techniques such as MRI and Computed Tomography (CT) [1]. Medical images are usually corrupted by noise during their acquisition and transmission [2]. The main objective of image de noising technique is to remove such noises while retaining as much as the important signal features [3]. Introductory section offers brief idea about different available de-noising scheme [4]. Ultrasound imaging is a widely used medical imaging procedure, because it is economical, comparatively safe, transferable and adaptable. One of its main shortcomings is the poor quality of images, which are affected by speckle noise. The existence of speckle is unattractive since it disgraces the image quality and affects the task of individual interpretation and diagnosis [5]. Accordingly, the speckle filtering is a central preprocessing step for feature extraction, analysis and recognition from medical imagery measurements. In this paper, methods of image de-noising based on discrete wavelet transform were proposed. However, we cannot obtain more high-frequency information only through discrete wavelet transform [6] [7]. An image's different scale detail information can be obtained through wavelet transform, but there will be some highfrequency information hidden in high-frequency sub-images of wavelet transform. If we decompose these highfrequency sub-images, we can obtained more image high-frequency information which can help us to enhance a US image effectively [8]. We should remove or reduce noise for the reason that there are lots of noises in highfrequency sub-images. For this we proposed a technique which is used to remove speckle noise from US image based on wavelet thresholding technique [9] [10].

The paper is arranged as follows: section I included the introduction and section II included the algorithm of the proposed scheme is explained with many details and Section III included the Evaluation Criteria and Section IV included Experimental result. Conclusions are shown in Section V.

1. Read an input noisy image $I(i, j)$

\section{Proposed Method}

2. Apply DWT to decompose noisy image up to 2 levels $(\mathrm{L}=2)$ to obtain sub bands, which are named as $\mathrm{LL}$, LH, HL, HH

3. Calculate threshold value for each sub-bands

4. Apply adaptive threshold in each (LH, HL,HH) sub-bands using given equations:

$$
\begin{array}{cr}
\text { Tnew }=\sigma \sqrt{2 \log M}, & \sigma=\frac{\operatorname{median}|Y(i, j)|}{0.6745} \\
\tilde{l}(i, j)=\left\{\begin{array}{cr}
(Y(i, j)-\mid \text { Tnew } \mid), & |Y(i, j)|>\text { Tnew } \\
0 & - \text { Tnew } \leq|Y(i, j)| \leq \text { Tnew } \\
(Y(i, j)+\mid \text { Tnew } \mid), & |Y(i, j)|<\text { Tnew }
\end{array}\right.
\end{array}
$$


5. Perform the inverse DWT to reconstruct the de-noised image.

6. Display the resultant image.

\section{Discrete Wavelet Transform}

The discrete wavelet transform employs multi-resolution filter banks and individual wavelet filters for the construction of multi-resolution time frequency plane and also for the analysis and reconstruction of signals. The DWT works on a whole original image and provide a different level of decomposition with coefficients block of image and the block of transformed coefficients are classified into types HH, HL, LH, and LL. These types are described as Diagonal, horizontal, vertical, and image approximation [6]. The DWT applied on an image is depicted by figure 1 .

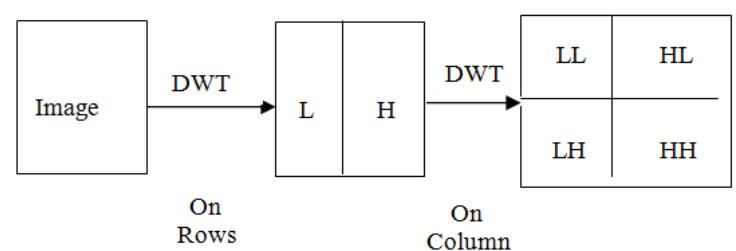

Fig.1: Application of DWT on image.

Suppose we have taken an original image and then we apply low pass filter and high pass filter on the rows. Since we are applying the wavelet transform in both dimensions, so we first apply on the rows and then columns. Whenever we apply low pass filter on rows to preserve the lowest discrete time frequency that is horizontal approximation and apply high pass filter to preserve the highest discrete time frequency that is horizontal detail. After getting the horizontal approximation we again apply low pass filter on column to get the approximate image (LL) and apply high pass filter on column to get the vertical details (LH). Similarly After getting the horizontal detail we again apply low pass filter on column to get the horizontal details (HL) and apply high pass filter on column to get the diagonal details (HH).The low-pass and high-pass decomposition filters are represented as $\mathrm{L}$ and $\mathrm{H}$, respectively. Applying in both horizontal and vertical directions to the original image $I(i, j)$, and next, recursively to the resulting gradually coefficients, we can obtain the following decomposition coefficients.

$$
\begin{gathered}
c A_{k+1}=\sum_{m=-\infty}^{+\infty} \sum_{n=-\infty}^{+\infty} L(2 i-m) L(2 j-n) A_{m, n} \\
c V_{k+1}=\sum_{m}^{+\infty}=-\infty \sum_{n=-\infty}^{+\infty} L(2 i-m) H(2 j-n) A_{m, n} \\
c H_{k+1}=\sum_{m=-\infty}^{+\infty} \sum_{n=-\infty}^{+\infty} H(2 i-m) L(2 j-n) A_{m, n} \\
c D_{k+1}=\sum_{m=-\infty}^{+\infty} \sum_{n=-\infty}^{+\infty} H(2 i-m) H(2 j-n) A_{m, n}
\end{gathered}
$$

The sub-bands are labeled by using following:

(1.1). LL $\left(C A_{k+1}\right)$ is the approximation image that indicate low-frequency component (LL) resulting from low-pass filtering in the vertical and horizontal directions.

(1.2). HL $\left(c V_{k+1}\right)$ represents the vertical details that results from vertical low-pass filtering and horizontal highpass filtering.

(1.3). LH ( $\left.c H_{k+1}\right)$ represents the horizontal details that results from horizontal low-pass filtering and vertical high-pass filtering.

(1.4). $\mathrm{HH}\left(c D_{k+1}\right)$ represents the diagonal details that results from high-pass filtering in both directions.

\section{Adaptive Threshold}

The discrete wavelet transform's smooth function can help us to reduce an image's noise, but it cannot meet our requirements. Still there is much noise in high frequency sub-images. If we enhance high frequency coefficients at this time, image detail information and noise are all enhanced. We reduce noises of highfrequency sub-images through the nonlinear method. Because the noise properties are different in different high frequency sub-images, So that different adaptive thresholds are used to reduce noise in different sub-images like diagonal, vertical and horizontal [8][9]. To set the soft threshold

$$
\text { Tnew }=\sigma \sqrt{2 \log M}
$$

Where $\mathrm{M}$ is the number of pixels in the image and $\sigma$ is the noise variance that is defined as: 


$$
\sigma=\frac{\text { median }|Y(i, j)|}{0.6745}
$$

Here $Y(i, j) \in H H$ sub-band coefficients those are obtained by applying the wavelet transform to the image. Now we adapt all sub-bands (except loest resolution subband) using following equation:

$$
\hat{Y}(i, j)=\left\{\begin{array}{lr}
(Y(i, j)-\mid \text { Tnew } \mid), & |Y(i, j)|>\text { Tnew } \\
0 & - \text { Tnew } \leq|Y(i, j)| \leq \text { Tnew } \\
(Y(i, j)+\mid \text { Tnew } \mid) . & |Y(i, j)|<\text { Tnew }
\end{array}\right.
$$

Where $\hat{Y}(i, j)$ is estimated sub-band.

\section{Evaluation Criteria}

To validate the efficiency of this method we have defined some statistical criteria of image performance. Additionally to subjective visual evaluation, it is desirable to present quantitative measure [11] [12] [13]. The parameters which are used in estimation of performance are SNR, PSNR, and RMSE.

\section{A. Signal to Noise Ratio (SNR)}

The Signal to Noise Ratio SNR is estimated by the following formula:

$$
S N R=\left[\frac{\sum_{1=1}^{\overline{\mathrm{M}}} \bar{\sum}_{\mathrm{j}=1}^{\mathrm{N}} \hat{l}\left(1_{1} \mathrm{j}\right)^{2}}{\left.\sum_{1=1}^{\mathrm{M}} \sum_{\mathrm{j}=1}^{\mathrm{N}} \hat{(}\left(\mathrm{I}_{s} \mathrm{j}\right)-\mathrm{I}\left(\mathrm{1}_{\mathrm{s}} \mathrm{j}\right)\right)^{2}}\right]
$$

Where I means original Image and $\widehat{I}$ means diffused Image. $\mathrm{M} \times \mathrm{N}$ is size of Image and i means row and $\mathrm{j}$ means columns.

\section{B. Peak Signal to Noise Ratio (PSNR)}

The Peak Signal to noise Ratio PSNR is estimated be the following formula [9]:

$$
\begin{array}{r}
\text { PSNR }=10 \log \left[\frac{255^{2}}{M S E}\right] \\
\text { Where, } \quad M S E=\left[\frac{\sum_{i=1}^{M} \Sigma_{j=1}^{\mathbb{N}}\left(\left[\left([i, j)-I([i, j))^{2}\right.\right.\right.}{M * N}\right]
\end{array}
$$

Where I means original Image and $\widehat{I}$ means diffused Image. $\mathrm{M} \times \mathrm{N}$ is size of Image and i means row and $\mathrm{j}$ means columns.

\section{Root Mean Square Error (RMSE)}

The Root Mean Square Error RMSE [9] calculated by the following equation:

$$
\text { RMSE }=\sqrt{\left(\frac{1}{\mathrm{MN}} \cdot \sum_{\mathrm{i}=1}^{\mathrm{M}} \sum_{\mathrm{j}=1}^{\mathrm{N}}\left(\mathrm{I}_{\mathrm{i}, \mathrm{j}}-\hat{\mathrm{I}}_{\mathrm{i}, \mathrm{j}}\right)^{2}\right)}
$$

\section{Experimental Result}

To validate the efficiency of our proposed method, the simulation study has been carried out using MATHLAB image processing Toolbox. One standard Medical image (Ultrasound Image) is selected for simulation study. Our Proposed algorithm has been applied to 2D ultrasound image with have been contaminated by multiplicative noise. Our proposed algorithm is compared with existing method which is shown in Table 1.

\begin{tabular}{|c|c|c|c|}
\hline Criteria & Adaptive Filter & Anisotropic Diffusion Filter & Proposed Filter \\
\hline SNR & 20.6741 & 20.9106 & 23.4105 \\
\hline PSNR & 29.1429 & 29.3351 & 31.8370 \\
\hline RMSE & 0.0654 & 0.0363 & 0.0330 \\
\hline
\end{tabular}

Table 1: Comparison of existing Adaptive and Anisotropic diffusion Filter and Proposed Method for Ultrasound image with Speckle noise. 
Visual Comparisons are given below:

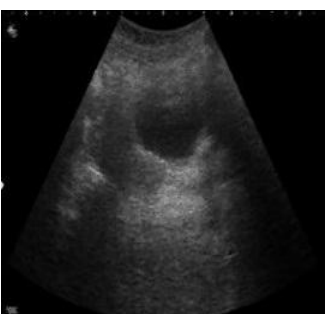

(a)

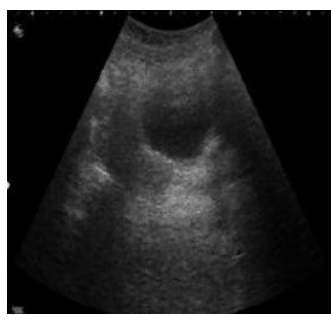

(b)

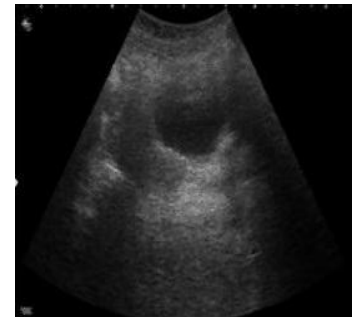

(c)

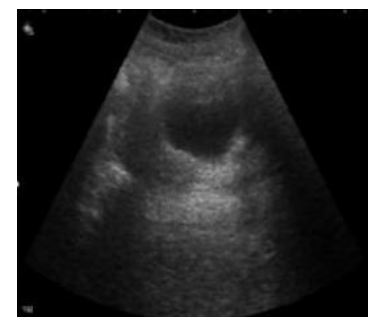

(d)

Fig 2: a). Original Noisy image. b). Adaptive filter image. c). Anisotropic diffusion filter image. d). Proposed filter image

\section{Conclusion}

Image de-noising has become a crucial step for correct diagnosis. The current need of healthcare industries is to preserve useful diagnostic information with minimum noise. In this paper, the proposed method's performance for denoising an image is evaluated both subjectively and objectively. The experimental results prove that the proposed method produces images which are cleaner and smoother and at the same time kept significant details, resulting in a clearer an appealing vision.

\section{References}

[1] R. C. Gonzalez and R. E. Woods, Digital Image Processing. Upper Saddle River, NJ: Prentice-Hall, 2001, pp. $572-585$.

[2] J. W. Goodman, "Some fundamental properties of speckle," J. Opt. Soc. Amer., vol. 66, no. 11, pp. 1145-1149, 1976.

[3] Pitas, and AN Venetsanopoulos, “Order Statistics in Digital Image Processing”, Proceed of the IEEE, Vol. 80, No. 12, Dec. 1992.

[4] "Image Independent Filter for Removal of Speckle Noise “. IJCSI International Journal of Computer Science Issues, Vol. 8, Issue 5, No 3, September 2011

[5] MICHAILOVICH O., TANNENBAUM A.: Despeckling of medical ultrasound images. IEEE Transactions on Ultrasonics,Ferroelectrics, and Frequency Control 53, 1 (2006), 64-78.

[6] Ms.Yamini S.Bute, Prof. R.W. Jasutkar, 2012, - Implementation of Discrete Wavelet Transform Processor For Image Compression International Journal of Computer Science and Network (IJCSN), Vol. 1.

[7] SU C., SEUL H.: A speckle reduction filter using waveletbased methods for medical imaging application. In Proceedings of the 23rd Annual International Conference of the IEEE Engineering in Medicine and Biology Society (2001), vol. 3,pp. $2480-2483$.

[8] S.Sudha, G.R. Suresh, and R.Sukanesh, "Wavelet Based Image Denoising using Adaptive Thresholding," International Conference on Computational Intelligence and Multimedia Applicatio, 2007, pp.296-300.

[9] S.G. Chang, B. Yu, and M. Vetterli, "Adaptive Wavelet Thresholding for Image Denoising and Compression," IEEE Trans. on Image Processing, Sept. 2000, vol. 9, No.9, pp. 15321546.

[10] Haryali Dhillon, Gagandeep Jindal, Akshay Girdhar, A Novel Threshold Technique for Eliminating Speckle Noise in Ultrasound Images, International Conference on Modelling, Simulation and Control, IPCSIT vol.10 (2011) IACSIT Press, Singapore.

[11] Z. Wang, A. Bovik, H. Sheikh, and E. Simoncelli, “Image quality assessment: From error measurement to structural similarity," IEEE Trans. Image Process., vol. 13, no. 4, pp. 600-612, Apr. 2004.

[12] Ahmet M. Eskicioglu, Paul S. Fisher, "Image Quality Measures and Their Performance" IEEE Transactions on Communication, Vol. 43, No. 12, pp. 2959-2965, December 1995.

[13] Zhou Wang, Alan C. Bovik, "A Universal Image Quality Index”, IEEE Signal Processing Letters, Vol. 9, No. 3, pp. 81-84, March 2002 . 